Original Article

\title{
Effect of Fasting On Cognitive Functioning In Healthy Young Adults
}

\author{
Shrunga M.S. ${ }^{1}$, Ayisha Shamooda ${ }^{2}$, Fathimath Shaniba ${ }^{3}$ \\ ${ }^{1}$ Lecturer, ${ }^{2,3}$ Interns, Nitte Institute of Speech and Hearing, M angalore, Karnataka, India - 575018. \\ *Corresponding Author : Shrunga M.S., Lecturer, Nitte Institute of Speech and Hearing, M angalore, Karnataka, India - 575018. \\ E-mail : msshrunga@gmail.com
}

Received

:20.03.2017

Review Completed : 22.04.2017

Accepted

:24.04.2017

Keywords: Cognition, Stroop Task, Executive Functions, Fasting

\begin{tabular}{|c|}
\hline Access this article online \\
\hline Quick Response Code \\
\hline
\end{tabular}

\begin{abstract}
Background and objective: Cognitive flexibility refers to the ability of the brain to switch from thinking about one concept to another simultaneously. The study was taken up to evaluate the effect of fasting on Cognitive Functioning In Healthy Young Adults.
\end{abstract}

Methodology: A total of 20 healthy young adults participated in the study. Paper Stroop Color Test was employed to assess inhibition and cognitive flexibility. Participants were given 4 cards (word card color card and color-word card) and were instructed to read the card as told by the clinician. The total duration taken to complete each task and incorrect responses for each task was noted. The same experiment was done in fasting and non-fasting condition for each participant.

Results: The results revealed no statistical significance between the 3 tasks between 2 conditions. Though our present study did not reveal statistical significance difference between 3 tasks and 2 conditions, it was found that the performance was better during the second condition i.e. after fasting condition in comparison to first condition i.e during fasting condition.

Conclusion: The study justifies these findings to be reflected due to fluid deprivation, change in life style and sleep wake up cycles with in turn effects cognitive functioning. The study also highlights on the effectiveness of stroop task in identifying the changes in cognitive function due to fasting.

\section{Introduction}

Cognition consists of set of abilities like attention, perception, memory, executive functions, processing speed etc. Any deficits or decline in these abilities will interfere with the daily communication processes which in turn may affect the quality of life of affected individuals. $M$ any studies have reported the importance of frontal lobe in executive functions, formation of strategies, rule following, cognitive flexibility, and evaluation of outcome ${ }^{[1]}$. Cognitive communication is a term, which encompasses many language skills, which are more complex than just retrieving words or forming sentences .It includes spoken and written expression and understanding, verbal and nonverbal aspects of communication such as tone of voice and facial expression. Cognitive-communication disorders are communication impairments resulting from underlying cognitive decline due to neurological impairment. These are difficulties in communicative competency (listening, speaking, reading, writing, conversation and social interaction) that result from underlying cognitive impairments (attention, memory, organization, information processing, problem solving, and executive functions). These disorders are distinct from other neurological communication disorders (e.g., aphasia, dysarthria etc.) and require specific techniques ${ }^{[2-4]}$. Frontal lobe plays a major role in higher level cognitive activity ${ }^{[5-7]}$. Cognitive flexibility has been described as the mental ability to switch between thinking about two different concepts, and to think about multiple concepts simultaneously. Functional Magnetic Resonance Imaging (fMRI) research has shown that specific brain regions are activated when a person engages in cognitive flexibility tasks. These regions include the Prefrontal Cortex (PFC),Basal ganglia, Anterior Cingulate Cortex (ACC), and Posterior Parietal Cortex (PPC). Measures for cognitive flexibility include the A-not-B task, Dimensional Change Card Sorting Task, M ultiple Classification Card Sorting Task, Wisconsin Card Sorting Task (WCST) , and The Stroop task. 
A study done using Go - No - Go test in cognitive flexibility showed that response time was slower but accuracy was not effected ${ }^{[8]}$. A study done using a computerized task similar to Wisconsin Card Sorting Task (WCST) to evaluate cognitive flexibility showed that fasting people had slower reaction time and they showed slowing at extra dimensional shift rather than intra dimensional shift ${ }^{[0]}$.

The stroop task tests psychological process and functions that effect cognition in normal populations. Some researchers suggests that individual who were fasting had poorer accuracy in stroop task for assessing executive functions ${ }^{[8,10]}$. Another study done using stroop task revealed that fasting has decreased speed of color naming ${ }^{[11]}$. One study that aimed to profile the effect of fasting during the Ramadan month on cognitive function in a group of healthy Muslim athletes revealed better responses for the tasks requiring sustained rapid responses in the morning, and the responses were found to decline in the late afternoon whereas performance in non-speed dependent accuracy measures was more flexible ${ }^{[12]}$. The consequences of the overnight and morning fast, particularly among the children who were nutritionally at risk, included slower stimulus discrimination, increased errors, and slower memory recall and these cognitive alterations are thought to result from a state of metabolic stress in which homeostatic mechanisms work to maintain circulating glucose concentrations ${ }^{[13]}$. Another study showed that hunger sensation affect working memory performance since it may distract attention of each individuals ${ }^{[14]}$. A study revealed no significant difference in working memory in people who habitually fasted for 24 hours ${ }^{[15]}$. Numerous investigations have been conducted on the impact of short-term fasting on cognition in healthy individuals. Some studies have suggested that fasting is associated with executive function deficits; however, findings have been inconsistency. Hence The present study is taken up an attempt to explore the effect of Fasting on Cognitive Functioning

\section{Aim of the study}

To evaluate the effect of Fasting on Cognitive Functioning In Healthy Young Adults

\section{Materials and Method Participants}

A total of 20 adults participated in the study .It consisted of 10 males and 10 females with an age range of 18 - 25 years. All the participants were proficient in English and were graduates and fasting individuals for a period of 30 days were included for the study. Subjects with Psychological or Neuromotor deficits were excluded from the study.

\section{Stimulus}

The stroop task consists of three cards: word card, color card and color-word card. The word card includes names of colors printed in black color i.e blue, red, green and yellow. Color card is a list of colored boxes in the same colors i.e blue, red, green and yellow. The color-word card is a list of color names printed in conflicting colors i.e blue written in red color, yellow written in blue color, etc.

\section{Procedure}

The study was carried out during the Ramadan fasting month. All the participants were examined at 2 different conditions; $3^{\text {rd }}$ week of fasting and two weeks after the end of Ramadan. Paper Stroop Color Test was employed to assess inhibition and cognitive flexibility. It consisted of three subtasks taking 45 seconds in/each one .In the first subtask (W )four basic color words including red, blue , yellow and green printed in black ink were presented as 25 words per page(4 pages).Subtask 2 (C) displayed solid color squares as 25 squares per page (4pages) and subtask 3 (CW) provided the participants with color words printed in an incongruent ink color presented as 25 words per page (4pages) (e.g., the word "blue" printed in red ink). In this task, participants were given all 4 pages with card W, followed by card $C$ and then card CW. The 4 cards were placed in front of the subjects on a table. After the subjects were given the 3 cards, they were instructed to read all the 100 words as fast as possible. For card $C$ the instructions were identical except that the participants were told to name the colors on the page. For card CW the participants were instructed name the ink colors regardless of the color words. The total duration taken to complete each task and incorrect responses for each task was noted. The same 
experiment was repeated two weeks after the end of Ramadan. The data was subjected to Paired t test using SPSS17.0.

\section{Results}

The results of the present study revealed no statistical significance $(p>0.05)$ between the 3 tasks between 2 conditions. Mean of total time taken to complete the task during and after Ramadan fasting across 3 tasks is depicted in fig 1 and Mean Incorrect response by the participants during and after Ramadan fasting across 3 tasks is represented in fig 2

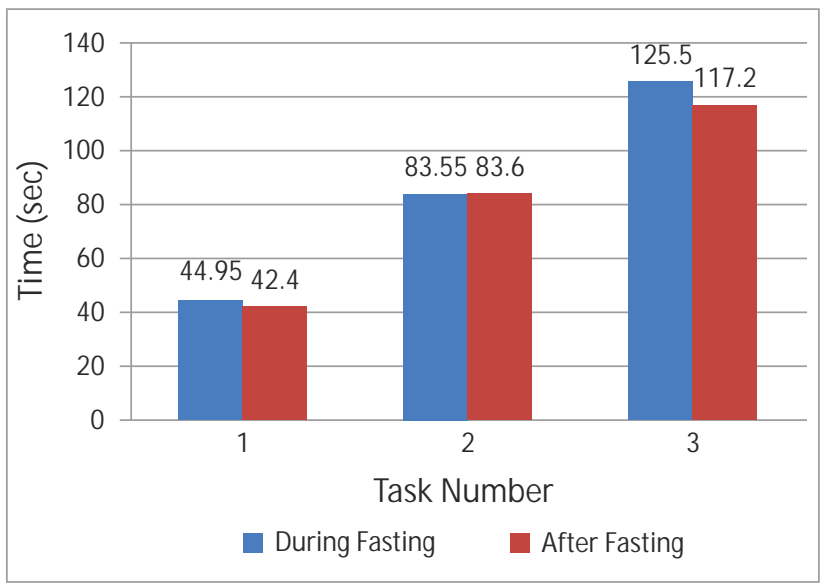

Fig 1 : Mean of total time taken to complete the task by the participants during and after Ramadan fasting across 3 tasks.

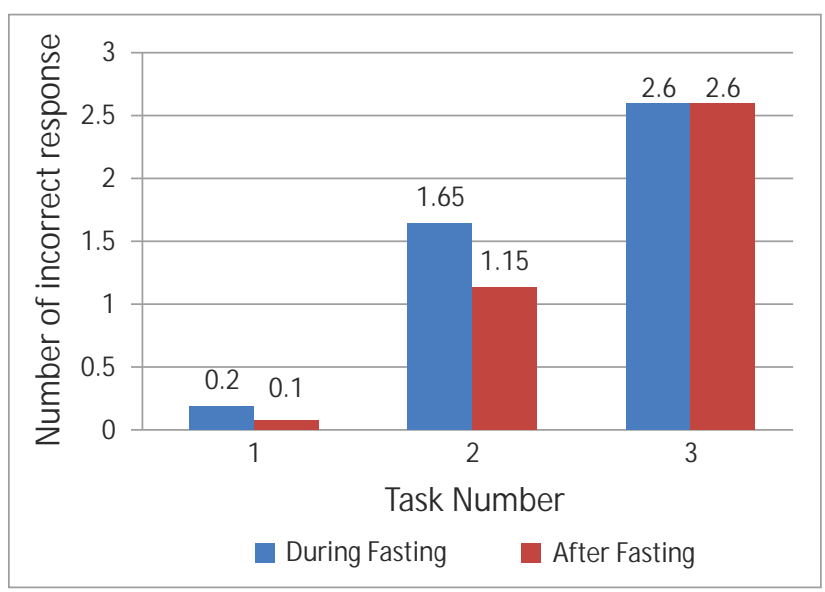

Fig 2 : Mean incorrect response by the participants during and after Ramadan fasting across 3 tasks.

\section{Discussion}

Our results are in agreement with the earlier findings on voluntary fluid deprivation ${ }^{[16]}$. Another reason which can be hypothesized for no significant difference in the findings between during fasting condition and after fasting condition is that the young healthy individuals would compensate for possible adverse effects of fluid deprivation during Ramadan by upgrading their mental effort and motivation ${ }^{[17]}$. It could also serve as a good explanation for unchanged objective performance in psychomotor tasks despite the worsened subjective feelings during Ramadan fasting ${ }^{[18]}$. Furthermore some studies addressed a deterioration of psychomotor performance at the beginning of Ramadan which was progressively reversed across the fasting period ${ }^{[19]}$. A similar pattern was also reported for subjective feeling variations during Ramadan ${ }^{[20]}$. Similar findings on memory and Stroop test were also reported from fasting ${ }^{[8]}$. Though our present study did not reveal statistical significance difference between 3 tasks and 2 conditions, it was found that the performance was better during the second condition i.e. after fasting condition in comparison to first condition i,e during fasting condition. This can be attributed to the fact that eating exclusively at nighttime imposes significant alterations on individuals' life style and sleep-wake cycles. The studies described that combination of changes in sleep-wake cycle, food and fluid intakes, and circadian rhythms was likely to affect mental, physical, and social performances ${ }^{[21]}$.Several subjective or objective assessments on the sleep patterns during Ramadan fasting reported sleep architecture alterations, delayed bedtime, and a significant reduction in nocturnal sleep ${ }^{[22,23]}$.Their findings showed that sleep loss might be responsible for excessive fatigue and reduced alertness in the daytime. Ramadan fasting also imposes deep impacts on the natural circadian rhythm through significant shifts in sleep patterns, body clock, and other physiological indices ${ }^{[21]}$ Irritability could be increased during Ramadan fasting which was attributed to reduction in sleep time or nicotine withdrawal ${ }^{[24]}$. Similarly, impairments in vigilance, memory, and continuous attention have been suggested in the course of Ramadan ${ }^{[25]}$.

\section{Conclusion}

The present study explores the strength of stroop task in identifying the changes in cognitive function due to fasting. The magnitude of the strength seems to depend upon factors like the time given for each task, and the parameters of analysis. Data acquisition on a larger sample 
with further controls may prove this task to be highly economical and effective in identifying changes related to healthy and pathological conditions.

\section{Acknowledgement}

I take the privilege to convey my sincere gratitude to The

\section{References}

1. Stuss DT, Eskes GA, Foster JK. Experimental neurophysiological studies of frontal lobe functions. Handbook of neuropsychology.1994.

2. Heilman KM , Safran A, Geschwind N. Closed head trauma and aphasia .J.neurol.neurosurg.psychiatry.1971; 34: 265-269.

3. Sarno MT. The nature of verbal impairment after closed head injury.J.Nerv.M ent.Dis.1980;168:2-19.

4. Ylvisaker M, Szekeres SF. M etacognitive and executive impairments in head injured children and adults.Top. Lang.Disord.1989;9:34-49.

5. Boller F, Grafman J (Eds.), Elsevier, Amsterdam. Handbook of neuropsychology .1994: 9

6. Nauta WJH. The problem of the frontal lobes: A reinterpretation. J. Psychiatr. Res.1971; 8:167-187

7. Stuss DT, Benson DF. The frontal lobes, Raven, New York 1986

8. Doniger GM , Simon ES, Zivotofsky AZ .Comprehensive assessment of cognitive sequelae of a complete 12 - 16 hour last. Behav.neurosci. 2006: 120: 804 - 816, doi: 10.1037/0735-7044.120.4.804.

9. Piech RM, Hampsire A, Owen AM, Pakinson JA.Modulation of cognitive flexibility by hunger and desire .Cogn.Emot.2008; 23:528540. Doi:10.1080/02699930802012153.

10. Owen AM .Fractionating attention control using event related $\mathrm{f} M \mathrm{RA}$ Cerebral cortex.2006; 12:1679-689.

11]Stewart SH, Samoluk SB .Effects of short term food deprivation and chronic dietary restrained on the selective processing of appetitive -related cues. Int. J. Eat. Disord.1997; 21: 129-135.

12. Tian HH, Aziz AR, Png W, Wahid M, Yeo D, Constance Png AL. Effects of fasting during Ramadan month on cognitive function in Muslim athletes. Asian.J.Sports. M ed.2011;2:145-153.

13. Ernesto Pollitt, Santiago Cueto, Enrique R Jacoby .Fasting and cognition in well and undernourished schoolchildren: a review of three experimental studies.Am.J.Clin.Nutr.1998; 67:779S-784S.

14. M ogg K, Bradley BP, Hyare H, Lee S. Selective attention to food-related stimuli in hunger are attentional biases specific to emotional and psychopathological states, or are they also found in normal drive
Director, Nitte Institute of Speech and Hearing and The Principal, Nitte Institute of Speech and Hearing for their constant support and encouragement. I would like to extend my gratitude to the Interns and the Participants for their support.

states?.Behav.Res.Ther.1998; 36: 227-237.

15. Dyna Rochmyangsih.J.Young.Investig.2009.

16. Szinnai et al Effect of water deprivation on cognitive motor performance in healthy men and women. Am. J. Physiol. Regul. Integr. Comp. Physiol .2005; 289: 275-280

17. Guti'errez A, Gonz'alez-Gross M, Delgado M, Castillo MJ .Three days fast in sportsmen decreases physical work capacity but not strength or perception-reaction time. Int. J. Sport. Nutr. Exerc. Metab. 2001; 11: 420-429.

18. Roky R, Iraki L, HajKhlifa R, Lakhdar Ghazal N. Daytime alertness, mood, psychomotor performances, and oral temperature during Ramadan intermittent fasting. Nutr.M etab.2000; 44:101-107.

19. Ghanouni P, M emari AM, Shayestehfar M, M oshayedi P, Gharibzadeh $\mathrm{S}$, Ziaee $\mathrm{V}$. Biological motion perception is affected by age and cognitive style in children aged 8-15.Neuro.Res.Int . 2015; Article ID 594042:6.

20. Lagarde D, Batejat D , Boussif M , Pradella S ,Girault S,Huppe M .Ramadan et vigilance . M 'edecine A'eronautique et Spatiale. 1996; 35: $175-182$.

21. Waterhouse J. Effects of Ramadan on physical performance chronobiological considerations. Br.J.Sports. Med. 2010; 44: 509-515.

22] M argolis, Reed. Effect of religious practices of Ramadan on sleep and perceived sleepiness of medical students. Teach.Learn.Med. 2004 Spring;16:145-9

23. Farooq, Herrera, Almudahka, Mansour, 2010. A Prospective Study of the Physiological and Neurobehavioral Effects of Ramadan Fasting in Preteen and Teenage Boys. J.Acad.Nutr.Diet. 2015 Jun; 115:889-97.

24. Kadri N, Tilane A, El Batal M, Taltit Y, Tahiri S M, Moussaoui D . Irritability during themonth of Ramadan. Psychosom.M ed. 2000; 62: 280-285

25. Dolu, Yuksek, Sizer, Alay .Arousal and continuous attention during Ramadan intermittent fasting. Basic.Clin.Physiol.Pharmacol.2007; 18: $315-22$. 